\title{
Network structure of brain atrophy in de novo Parkinson's disease
}

\section{Yashar Zeighami ${ }^{1}$, Miguel Ulla ${ }^{1,2}$, Yasser Iturria-Medina ${ }^{1}$, Mahsa Dadar ${ }^{1}$, Yu Zhang ${ }^{1}$ Kevin Michel-Herve Larcher ${ }^{1}$, Vladimir Fonov ${ }^{1}$, Alan C Evans ${ }^{1}$, D Louis Collins ${ }^{1}$, Alain Dagher ${ }^{1 *}$}
${ }^{1}$ McConnell Brain Imaging Centre, Montreal Neurological Institute, McGill University, Montreal, Canada; ${ }^{2}$ Service de Neurologie A, CHU Clermont-Ferrand, Clermont- Ferrand, France

${ }^{*}$ For correspondence: alain. dagher@mcgill.ca

Competing interests: The authors declare that no competing interests exist.

Funding: See page 16

Received: 30 April 2015

Accepted: 05 September 2015

Published: 07 September 2015

Reviewing editor: David C Van Essen, Washington University in St Louis, United States

(c) Copyright Zeighami et al. This article is distributed under the terms of the Creative Commons Attribution License, which permits unrestricted use and redistribution provided that the original author and source are credited.

\section{Introduction}

Parkinson's disease (PD) is the second most common neurodegenerative illness. Its clinical hallmarks are due to loss of dopamine neurons in the substantia nigra (SN); however, post-mortem studies have shown that PD pathology affects several other brain areas (Goedert et al., 2013). Surprisingly, however, magnetic resonance imaging (MRI) studies have failed to consistently demonstrate regional brain atrophy at least in the earlier stages of the disease. This is possibly due to studies consisting mostly of small numbers of subjects from single centers and the use of methodological tools that are relatively insensitive to subcortical atrophy, coupled with univariate methods that suffer from lack of statistical power.

The distribution of Lewy body pathology in post-mortem samples led Braak et al. to propose that a disease process spreads from the brainstem to subcortical areas and then to the cerebral hemispheres along neuronal pathways (Braak et al., 2003, 2006; Goedert et al., 2013). More generally, the network-spread hypothesis suggests that all neurodegenerative diseases target spatially distributed intrinsic brain networks (Warren et al., 2013). At a molecular level, neurodegenerative diseases are now thought to involve prion-like spread of toxic misfolded protein aggregates (Frost and Diamond, 2010; Jucker and Walker, 2013). Alpha-synuclein fibrils, implicated in PD pathogenesis, have recently been shown to spread from cell to cell via neuronal pathways after inoculation in mouse brain (Luk et al., 2012; Masuda-Suzukake et al., 2013).

Neuroimaging studies have revealed that the spatiotemporal organization of the brain takes the form of intrinsic connectivity networks (ICNs) (Fox and Raichle, 2007; Smith et al., 2009), which are consistent across studies, in test-retest analysis, and during both rest and task states (Damoiseaux et al., 2006; Smith et al., 2009; Zuo et al., 2010). Intrinsic networks have also been thought to be potential targets 
eLife digest Although Parkinson's disease is the second most common neurodegenerative disorder, its cause is not known and there is no cure. The symptoms of Parkinson's disease, which include tremor and slowing of voluntary movements, get progressively worse over time. The numbers of neurons in certain brain regions also decrease, causing those parts of the brain to shrink; this is known as 'atrophy'. However, no conclusive signs of atrophy have been found in the brains of people in the early stages of the disease.

One theory suggests that Parkinson's disease is caused by a toxic protein that is able to spread from neuron to neuron. Recent advances in brain imaging have made it possible to map networks in the living human brain-the so-called brain connectome. These networks could form the 'highways' through which a disease-causing agent might spread.

The Parkinson's Progression Markers Initiative (PPMI) is a large study that collects data from hundreds of people in an effort to identify the causes of Parkinson's disease. Zeighami et al. have now analyzed MRI scans that were collected as part of this initiative, which show the structure of the brains of 230 people in the early stages of Parkinson's disease. Comparing these scans to those from age-matched healthy individuals allowed Zeighami et al. to identify the set of brain regions that show atrophy in the early stages of Parkinson's disease. These regions correspond to a normal brain network, and the relative extent of atrophy in each brain region supports the theory that the disease spreads through the connectome.

The patients who were enrolled in this study will continue to be evaluated on a yearly basis. Zeighami et al. plan to continue mapping how the disease progresses throughout the brain and to relate this to the development of new symptoms of Parkinson's disease.

DOI: 10.7554/eLife.08440.002

for $A D$ since the earliest reports of default mode network dysfunction in the disease (Greicius et al., 2004), and analysis of large MRI data sets in AD and other dementias have supported the network-vulnerability hypothesis (Seeley et al., 2009; Raj et al., 2012; Zhou et al., 2012); however, it has yet to be tested in PD. Here, we analyzed data from newly diagnosed PD patients $(n=232)$ and an age-matched control group ( $n=117$ ) obtained from the Parkinson's Progression Markers Initiative (PPMI) database (www.ppmi-info. org/data), an observational, multicenter longitudinal study designed to identify PD progression biomarkers (Marek et al., 2011). We used deformation-based morphometry (DBM) and tensor probabilistic independent component analysis (ICA) to identify brain regions demonstrating atrophy in early PD.

We also sought to provide support for the network-spread hypothesis in PD by showing that the disease, in humans, targets intrinsic brain networks. We compared the set of atrophic regions in PD patients to ICNs from young healthy subjects and tested them for spatial overlap. To further investigate the model of disease propagation through brain networks, we used resting state functional MRI (fMRI) and diffusion-weighted MRI (DW-MRI) of healthy subjects to define the normal brain connectome and determined whether the pattern of atrophy in PD was compatible with spread via this network from a presumed disease epicenter in the SN.

\section{Results}

\section{ICA detects a PD-specific pattern of deformation}

DBM was used as the measure of local brain atrophy. It is a measure of the change in the shape of each voxel that results from applying non-linear spatial normalization of the entire brain to a standard template (Aubert-Broche et al., 2013). For each subject, we obtained one parametric image of MRIdetermined voxel-wise difference in volume, compared to the template brain. ICA was conducted on these DBM images using FSL MELODIC software (Beckmann and Smith, 2004). No constraint was imposed on the number of components, and probabilistic ICA estimated 30 independent components of deformation in the PPMI data set (PD patients and controls combined). Each ICA component consists of a spatial map and the average deformation value in that map for each subject.

In each of these 30 independent components, the average deformation between PD and control groups was compared using an unpaired t-test. PD patients had significantly lower DBM values in one and only one of the 30 deformation components $(p=0.0001$ uncorrected, $p=0.003$ with Bonferroni 
correction). The next spatial component in terms of statistical significance ( $p=0.06$, uncorrected) consisted of cerebellar areas VIIIA, VIIB, CrusIl, and IX known for their involvement in motor and executive function (Stoodley and Schmahmann, 2009). None of the other components demonstrated a difference between PD and controls ( $p>0.05$ uncorrected for all remaining components). We will call the deformation pattern showing a group difference the PD-ICA network from this point on. Ten of the other ICA components demonstrated an effect of age in the entire group. In three of these, there was a positive correlation between the component expression and age (meaning increased volume with age) and all three consisted of ventricle or cerebrospinal fluid space enlargement (Figure 1-figure supplement 1). The other seven age-related components demonstrated a negative correlation (volume loss with age) and consisted of areas of gray or white matter (Figure 1-figure supplement 2). The PD-ICA network also demonstrated greater atrophy with increasing age in both PD patients $\left(r=-0.38, p<10^{-9}\right)$ and controls $\left(r=-0.53, p<10^{-9}\right)$.

\section{Spatial analysis of PD-ICA network}

Each spatial map was converted to a z-statistic image via a normalized mixture model and then thresholded at $z \geq 3$. Regions were identified using the atlases of Talairach and Tournoux (1988) and Mai et al. (2003). The PD-ICA network includes all components of the basal ganglia (substantia nigra, subthalamic nucleus, nucleus accumbens, putamen, caudate nucleus, and internal and external globus pallidus), the pedunculopontine nucleus, basal forebrain, including bed nucleus of the stria terminalis and an area containing the nucleus basalis of Meynert, the hypothalamus, amygdala, hippocampus, parahippocampal gyrus, and two thalamic regions, the ventrolateral nucleus and pulvinar. Cortical regions in this network are the insula, occipital cortex Brodmann area 19, superior temporal gyrus, rostral anterior cingulate cortex, premotor and supplementary areas, and parts of lateral prefrontal cortex (Figure 1, Tables 1, 2; see also Figure 1-figure supplement 4).

\section{Clinical correlation}

To confirm that the PD-ICA network identified above was disease-related, we compared individual deformation values in the network to measures of disease severity. The two clinical measures used were the striatum binding ratio (SBR) measured with single photon emission computed tomography (SPECT) using the tracer [ ${ }^{123}$ ] FP-CIT (Booij and Knol, 2007) to measure dopamine nerve terminal density, and the score on the Movement Disorder Society revised Unified Parkinson's Disease Rating Scale (UPDRS) part III (Goetz et al., 2008), an objective measure of motor disability. For SBR, we used the average value of left and right putamen. There was a significant correlation between individual SBR and DBM values in the PD-ICA network in the PD group ( $r=0.23, p<0.0005$, Figure 2). This shows that the greater the loss of dopamine nerve terminals, the greater the volume loss in the PDICA network. There was also a significant correlation between these two measures in the control group ( $r=0.33, p<0.0005)$.

There was a significant correlation between DBM values within the PD-ICA network and UPDRS III in the PD patients $(r=-0.22, p<0.001$; Figure 2). SBR was not significantly correlated with age in the PD subjects $(r=-0.10, p=0.12)$ but it was in controls $(r=-0.35, p<0.0001)$. Also, in PD subjects, SBR was significantly correlated with UPDRS III $(r=0.20, p=0.002)$. We also tested whether UPDRS III was correlated with DBM values obtained from any one of the other 29 ICA components. There was only one other component marginally correlated with disease severity $(r=-0.2, p=0.048$, Bonferroni corrected) consisting of the previously mentioned cerebellar network, areas VIIIA, VIIIB, CrusII, and IX. Because age, UPDRS III, and SBR all correlated with PD-ICA DBM, we performed multiple linear regressions. In the PD group, the model (PD-ICA $1+$ Age + UPDRS III + SBR) showed an effect of age $\left(p=2.4 \mathrm{e}^{-08}\right)$, UPDRS III $(p=0.06)$ and SBR $(p=0.01)$. In the controls, the model (PD-ICA $1+$ Age + SBR) demonstrated an effect of age $\left(p=4.7 e^{-08}\right)$ but not SBR $(p=0.17)$.

Finally, multivariate analysis was used to look for an effect of scanning site. For each obtained DBM-network, we applied the model: DBM Group (PD/Control) + Age + Gender + Site. There was no significant effect of site after correcting for multiple comparisons $(p>0.1)$.

\section{Comparing disease-related atrophy to functional networks in health}

We next tested the hypothesis that the PD-ICA deformation network represents an intrinsic functional network. We compared the PD-ICA network as well as the other 29 ICA maps obtained from the DBM 


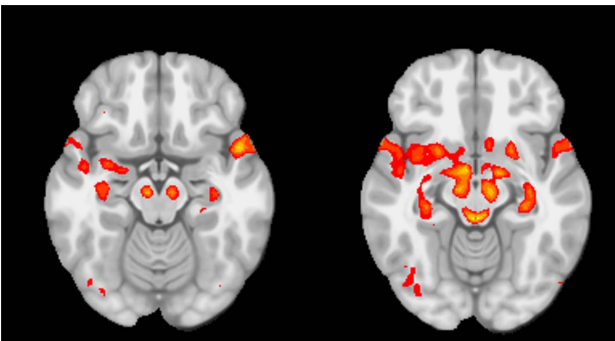

$\mathbf{R}$
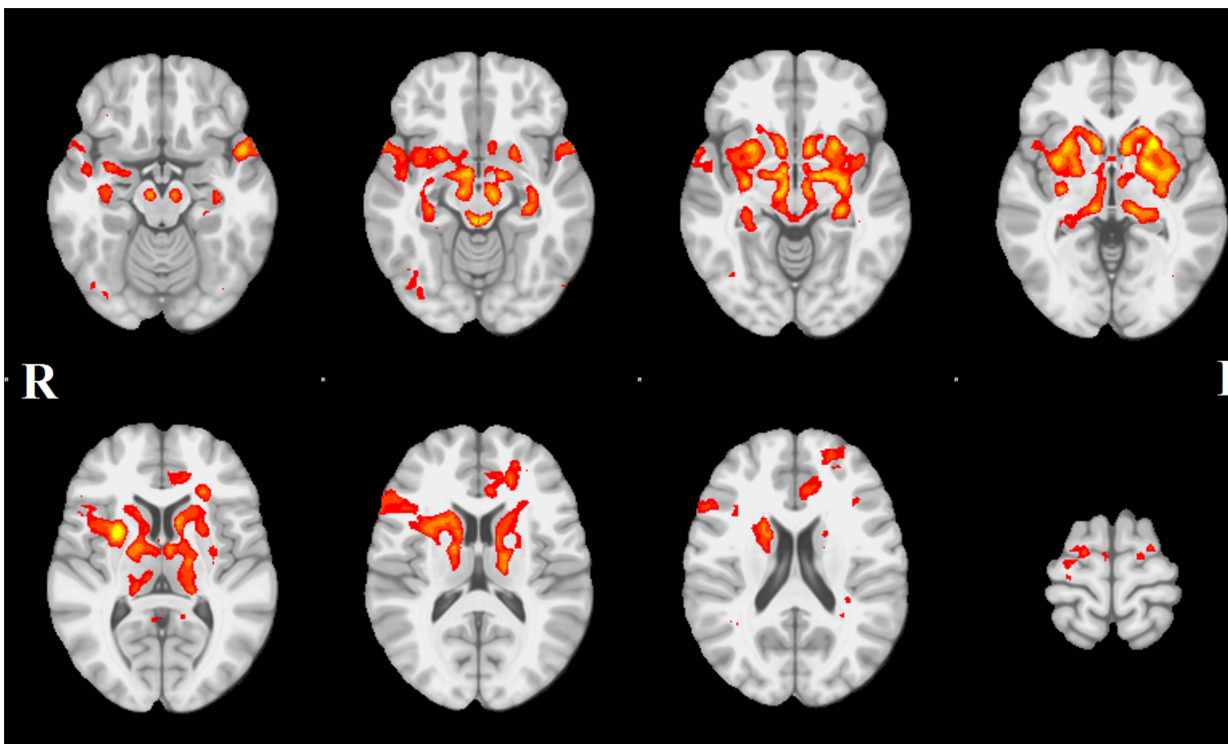

$\mathbf{L}$

Figure 1. Distribution of atrophy in Parkinson's disease. This image displays the only one of the 30 independent component analysis (ICA) networks showing a significant difference between Parkinson's disease (PD) and Controls $(p=0.003$ after correction for multiple comparison). The ICA spatial map was converted to a z-statistic image via a normalized mixture-model fit and then thresholded at $z=3$. Selected sections in Montreal Neurological Institute (MNI) space at coordinates $z=-16, z=-12, z=-7, z=-2, z=8, z=14, z=20, z=70$. See Tables 1, 2 for anatomical localization. Note that the value at each voxel is the $z$-score of the ICA component, not the group difference.

DOI: 10.7554/eLife.08440.003

The following figure supplements are available for figure 1:

Figure supplement 1. Networks with positive correlation between average deformation and age. DOI: 10.7554/eLife.08440.004

Figure supplement 2. Networks with negative correlation between average deformation and age. DOI: 10.7554/eLife.08440.005

Figure supplement 3. Overlapping areas between the PD-ICA network obtained from the PPMI data set and regions from Masuda-Suzukake et al. (2013).

DOI: 10.7554/eLife.08440.006

Figure supplement 4. Voxel-wise difference in atrophy between PD and controls.

DOI: 10.7554/eLife.08440.007

ICA analysis to intrinsic brain networks in healthy brain. In order to increase confidence in the results, we determined normal intrinsic brain networks in several different ways:

1. A seed-based functional connectivity map obtained from resting-state fMRI (rsfMRI) in 51 healthy volunteers with an a priori region of interest in the SN.

2. Two sets of 70 and 100 typical functional resting-state networks from healthy volunteers previously identified with MELODIC ICA by Smith et al. (2009) and Smith et al. (2013).

First, we compared the resting-state SN seed-based map (Figure 3B) with all 30 structural maps obtained from the PPMI ICA analysis. Only two ICA networks passed the threshold of $|r|>0.25$. These two networks correspond to very similar subcortical networks in basal ganglia and brainstem, one of which is mostly white matter areas $(r=0.36)$, while the other is mostly gray matter areas $(r=0.30)$. The latter is the aforementioned PD-ICA network in which atrophy correlated with disease severity (Figure 3-figure supplement 1).

Then, we compared the PD-ICA network to the 70 rsfMRI ICNs in normal brain provided by Smith et al. (2009). One of the 70 networks passed the threshold (with $r=0.32$ ). This network has been related to reward tasks, interoceptive functions, and motor/sensory processing (Figure 3C). 
Table 1. PD-ICA subcortical anatomical areas

\begin{tabular}{|c|c|c|c|}
\hline Brain area & Sub-areas & Z-score L/R & Peak coordinate L/R \\
\hline Entorhinal cortex & - & na $(2.94) / 3.1$ & $-20,-7,-32 / 19,-4,-34$ \\
\hline Claustrum & - & $\mathrm{na} / 4.3$ & $\mathrm{na} / 36,0,-21$ \\
\hline Amygdala & Basolateral & $3.8 / 4.1$ & $-23,-5,-21 / 22,-4,-20$ \\
\hline Hippocampus & Dentate Gyrus & $4.7 / 4.2$ & $-34,-18,-14 / 34,-15,-17$ \\
\hline Substantia nigra & - & $5 / 4.9$ & $-8,-18,-15 / 7,-17,-15$ \\
\hline Periaqueductal gray & - & $5.6 / 5.4$ & $-3,-34,-12 / 3,-33,-12$ \\
\hline Pedunculopontine nucleus & - & $4.7 / 4.6$ & $-6,-30,-11 / 6,-29,-11$ \\
\hline Hypothalamus & - & $3.4 / 4.2$ & $-5,-3,-11 / 4,-3,-11$ \\
\hline Hippocampus & CA1, CA2, CA3 & $5.3 / 4.5$ & $-30,-27,-10 / 31,-27,-11$ \\
\hline Subthalamic nucleus & - & $5.2 / 5.2$ & $-8,-16,-10 / 9,-16,-10$ \\
\hline Nucleus accumbens & - & $5 / 4.9$ & $-9,11,-9 / 8,11,-8$ \\
\hline Basal forebrain & BNST & $3.3 / 3.6$ & $-6,4,-8 / 9,3,-10$ \\
\hline Basal forebrain & Extended amygdala & $6 / 5.8$ & $-16,-6,-8 / 10,-6,-8$ \\
\hline Basal forebrain & Substantia innominata & $3.6 / 5.1$ & $-8,0,-8 / 8,0,-8$ \\
\hline Putamen & Anterior putamen & $5.6 / 4.8$ & $-25,11,-5 / 25,11,-5$ \\
\hline Putamen & Posterior putamen & $6.1 / 4.8$ & $-30,-12,-6 / 31,-15,-2$ \\
\hline Globus pallidus & Internal + external & $5.7 / 4.7$ & $-20,1,-1 / 21,-3,-3$ \\
\hline Caudate nucleus & Head & $8.2 / 6.2$ & $-10,12,4 / 10,10,2$ \\
\hline Pulvinar & Medial/Lateral & $5.3 / 4.5$ & $-19,-31,5 / 11,-26,-4$ \\
\hline Thalamus & Ventrolateral/Ventroanterior & $5.3 / 3.6$ & $-17,-14,11 / 14,-14,12$ \\
\hline Caudate & Body & $4 / 4.8$ & $-15,11,12 / 17,10,15$ \\
\hline
\end{tabular}

List of subcortical regions belonging to the PD-ICA network and their peak z-scores. (BA: Brodmann area, na: not applicable, BNST: bed nucleus of the stria terminalis, ICA: independent component analysis, PD: Parkinson's disease). DOI: 10.7554/eLife.08440.008

We assessed statistical significance by generating 1000 permutations of each of the 70 ICNs by reassigning the coordinates of each voxel randomly (Figure 3-figure supplement 2). We then repeated this comparison using a finer decomposition of 100 resting-state ICNs from the Human Connectome Project (HCP) (Smith et al., 2013) using MELODIC. Four components showed spatial

Table 2. PD-ICA cortical anatomical areas

\begin{tabular}{llll} 
Brain area & Sub-areas & Z-score L/R & Peak coordinate L/R \\
\hline Superior temporal gyrus & Temporal pole BA 38 & $5.6 / 3.6$ & $-50,11,-18 / 50,10,-12$ \\
\hline Occipital lobe & BA 19 & $3.1 / 3.4$ & $-39,-77,-18 / 35,-79,-14$ \\
\hline Insula & Mid-insula & $4.5 / 4.6$ & $-39,0,-5 / 38,5,-2$ \\
\hline Inferior frontal gyrus & BA 45 & $3.4 / 4.4$ & $-38,26,19 / 53,26,15$ \\
\hline Anterior cingulate cortex & Rostral ACC & $4.3 /$ na & $-6,31,18 /$ na \\
\hline Middle frontal gyrus & DLPFC BA 9/46 & $4.1 /$ na & $-22,51,19 /$ na \\
\hline Superior frontal gyrus & BA 6 & $4 / 3.7$ & $-18,-10,66 / 23,-10,54$ \\
\hline Supplementary motor area & - & na/3.4 & na/5, -12, 67
\end{tabular}

List of cortical regions belonging to the PD-ICA network and their peak z-scores. (BA: Brodmann area, na: not applicable, ACC: anterior cingulate cortex, DLPFC: dorsolateral prefrontal cortex, ICA: independent component analysis, PD: Parkinson's disease).

DOI: 10.7554/eLife.08440.009 

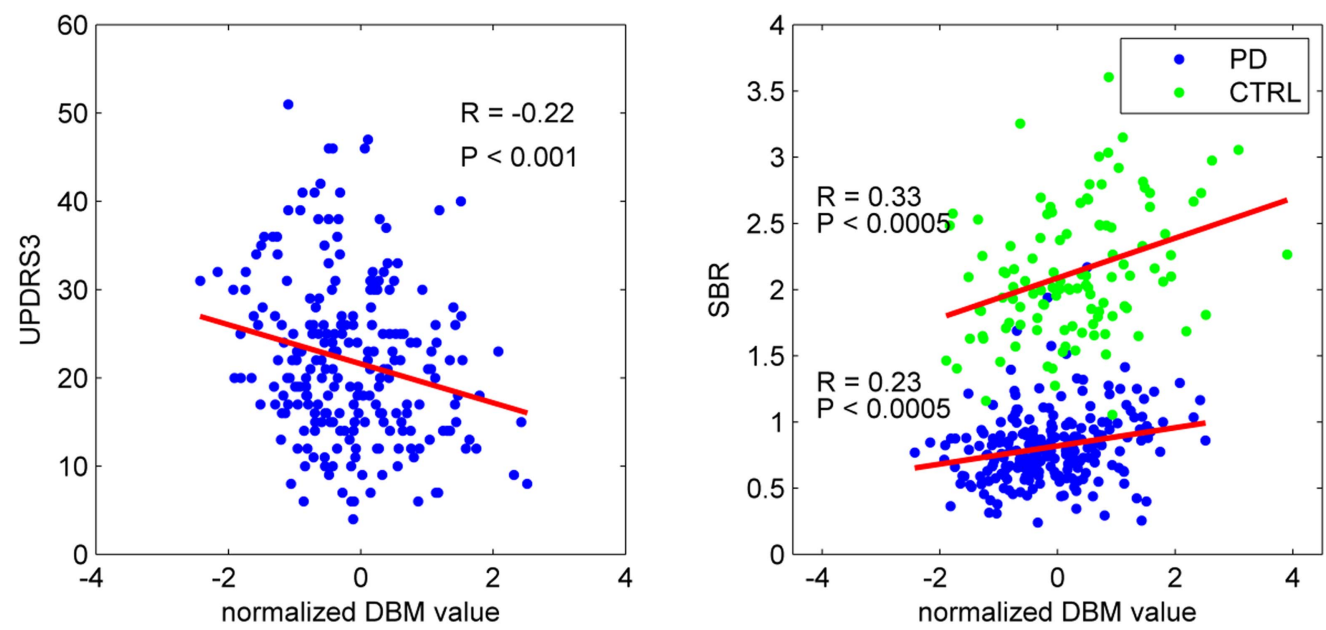

Figure 2. PD-ICA network, dopamine denervation, and severity of disease. Left: Unified Parkinson's Disease Rating Scale (UPDRS) part III (a measure of motor function and disease severity-higher value means more severe disease) was significantly correlated with the degree of atrophy in the network $(r=-0.22, p<0.001)$. Right: plot of [123l]FP-CIT striatum binding ratio (SBR) vs deformation value in the PD-ICA (Figure 1). Correlation: $r=0.23, p<0.0005$ for PD patients, and $r=0.33, p<0.0005$ for age-matched controls.

DOI: 10.7554/eLife.08440.010

overlap to the PD-ICA network using spatial correlation (Figure 3-figure supplement 3). The mean fMRI time series from these components were then used to determine whether they themselves belonged to one larger ICN. These time series demonstrated significant inter-correlation $(p<0.0016$ by permutation testing). Finally, hierarchical clustering (Smith et al., 2013) confirmed that all four components clustered together.

We also compared the PD-ICA network to a map of regions responding to stimulus value during fMRI experiments as identified by meta-analysis (Bartra et al., 2013). In these experiments, subjects typically evaluated an offered item (say a food) and experimenters identified brain regions where the fMRI signal tracked subjective value (e.g., willingness to pay for the item). The premise is that PD may affect dopamine projection sites that encode aspects of stimulus value. Only one of the 30 networks passed the threshold ( $r=0.28$ ), namely the aforementioned PD-ICA network (Figure 3D).

In summary, the PD-ICA network exhibited significant spatial overlap with presumed intrinsic brain networks determined by three different methods.

\section{Testing the propagation model}

The sequential propagation model predicts that the spatial progression of the disease process will be determined by brain network topology. Connectivity between any region and the presumed disease epicenter will determine how severely it is affected. Here, we evaluated this assumption by exploring whether the gray matter atrophy patterns observed in PD patients could be explained by functional and geodesic distance (i.e., the number of edges separating two nodes in a graph) to the hypothetical pathogenic epicenter. We chose to use the SN as the epicenter based on known PD pathology. Note that the SN is unlikely to be the first affected site in the central nervous system (CNS) (Braak et al., 2003, 2004); however, we postulate that it is likely to function as a source for propagation to the supratentorial brain. Network connectivity in health can be defined functionally, using rsfMRI, or structurally, using DW-MRI. The influence of disease on each node can be estimated from the statistical difference in deformation between PD and control groups. We parcellated the brain into 112 regions of interest (ROls, Figure 4-figure supplement 1) and computed the degree of deformation in each region. We also generated two connectomes from these ROI using rsfMRI and DW-MRI data from two different pools of healthy subjects. The connection strength between each pair of regions was computed as described in the 'Materials and methods'. 

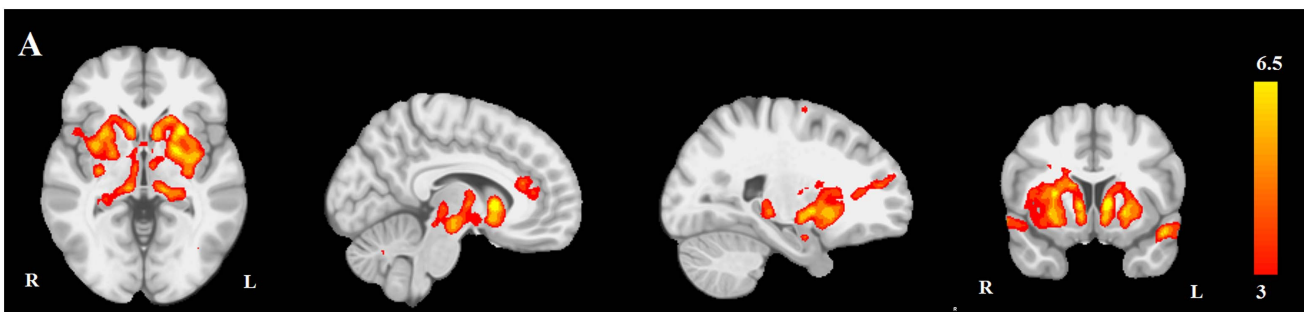

B
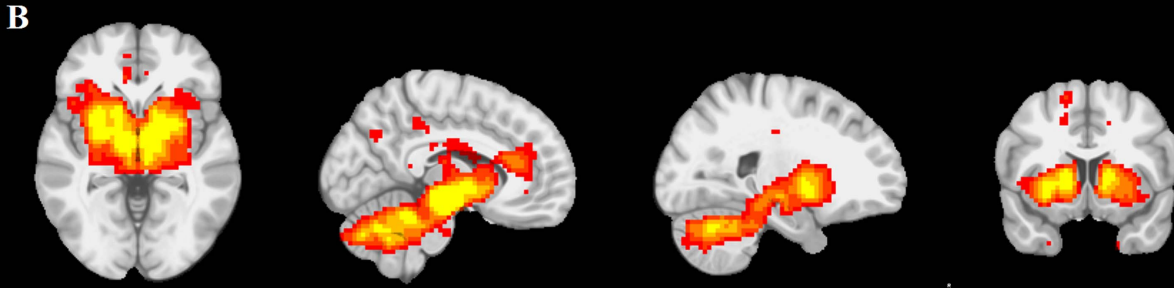

10
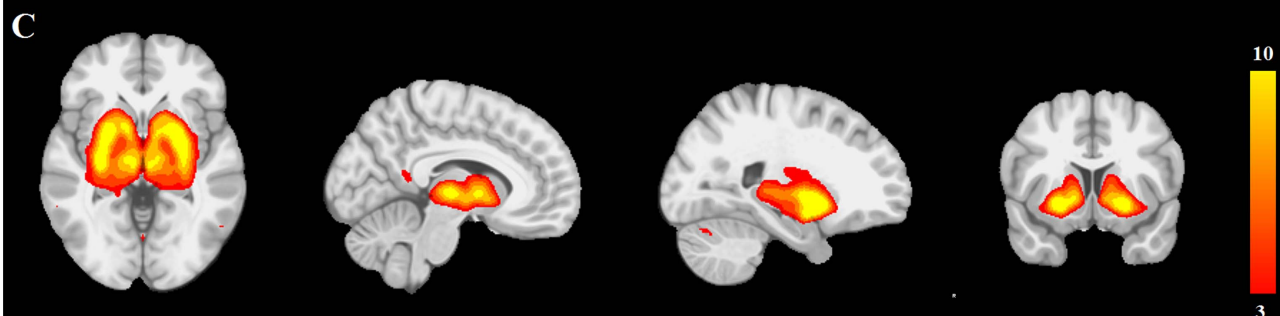

D
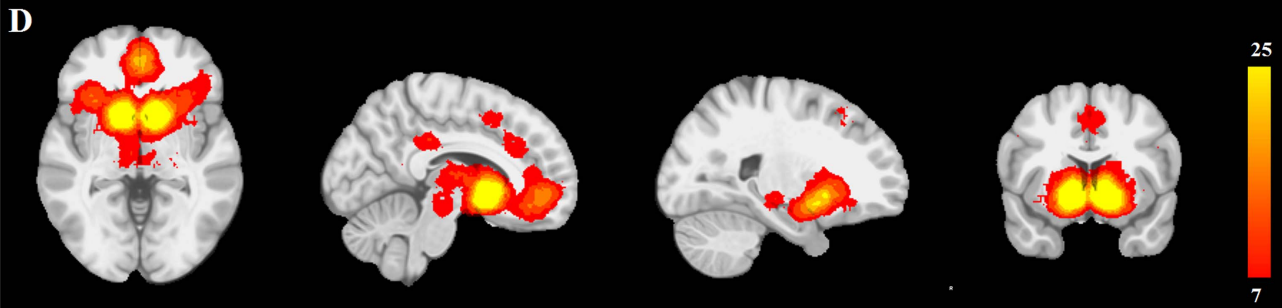

Figure 3. PD atrophy resembles normal intrinsic connectivity networks. Selected sections for (A) PD-ICA network from the Parkinson's Progression Markers Initiative (PPMI) data set thresholded at $z=3$. (B) Seed-based resting-state functional MRI (fMRI) connectivity with substantia nigra as a priori seed. (C) Intrinsic connectivity network (ICN) correlated with PD-ICA from Smith et al. (2009). (D) Regions responding to stimulus value during fMRI (metaanalysis of Bartra et al., 2013) (Selected slices in MNI space $z=-2, x=-8, x=-23, y=10$.)

DOI: 10.7554/eLife.08440.011

The following figure supplements are available for figure 3:

Figure supplement 1. Selected slices for seed-based resting-state fMRI analysis results with $\mathrm{SN}$ as a priori seed (top), PD-ICA network from the PPMI data set (middle), ICA network consisting of white matter areas in basal ganglia and cerebellum (bottom).

DOI: 10.7554/eLife.08440.012

Figure supplement 2. The correlation between the PD-ICA network and the 70 ICNs from Smith et al. (2009) is displayed in red.

DOI: 10.7554/eLife.08440.013

Figure supplement 3. Correspondence between the PD-ICA network and resting-state networks (RSN) from the Human Connectome Project (HCP).

DOI: 10.7554/eLife.08440.014

There was a significant correlation between resting-state functional connectivity of each node with SN (in healthy brain) and the PD-related deformation (PD minus control t-score), $(r=0.40, p<0.0001)$. We repeated the same analysis controlling for spatial proximity between each region and $\mathrm{SN}$ by 
entering Euclidean distance as a covariate. The correlation was unchanged $(r=0.38, p<0.0001)$, suggesting that the relationship cannot be explained by spatial proximity. The correlation implies that higher functional connection between a given region and SN is related to higher PD-related atrophy in that region (Figure 4). When using an anatomical measure of connectivity (DW-MRI) (Figure 4), we also observed a significant relationship between the level of atrophy of each region and its geodesic distance to the SN $(r=-0.28, p<0.003)$. These results were not different after controlling for Euclidean distance to $\mathrm{SN}(r=-0.25, p<0.005)$. Finally, we repeated this analysis using every $\mathrm{ROI}$ as a potential disease propagator and found that $\mathrm{SN}$ was the likeliest disease propagator when using the rsfMRI connectome (Table 3). However, the red nucleus $(r=0.28)$ and subthalamic nucleus $(r=0.28)$ were also identified as potential propagators. Repeating this analysis using a tractography-derived connectome also revealed that the SN was one of the likeliest propagators, but numerous cerebellar regions also emerged as potential propagators (Figure 4-source data 2). This may be due to difficulty in accurate identification of the targets of brainstem white matter tracts using DW-MRI (Ford et alo, 2013).

\section{Discussion}

\section{Pattern of atrophy in PD}

The combination of DBM and ICA of an unprecedentedly large set of MRI data at a magnetic field strength of $3 \mathrm{~T}$ allowed us to map out the brain regions affected in de novo PD. Patients included in this study were diagnosed on average 7 months prior to the investigation and had no evidence of dementia (Table 4). Most MRI studies to date using T1-weighted images in PD have reported normal
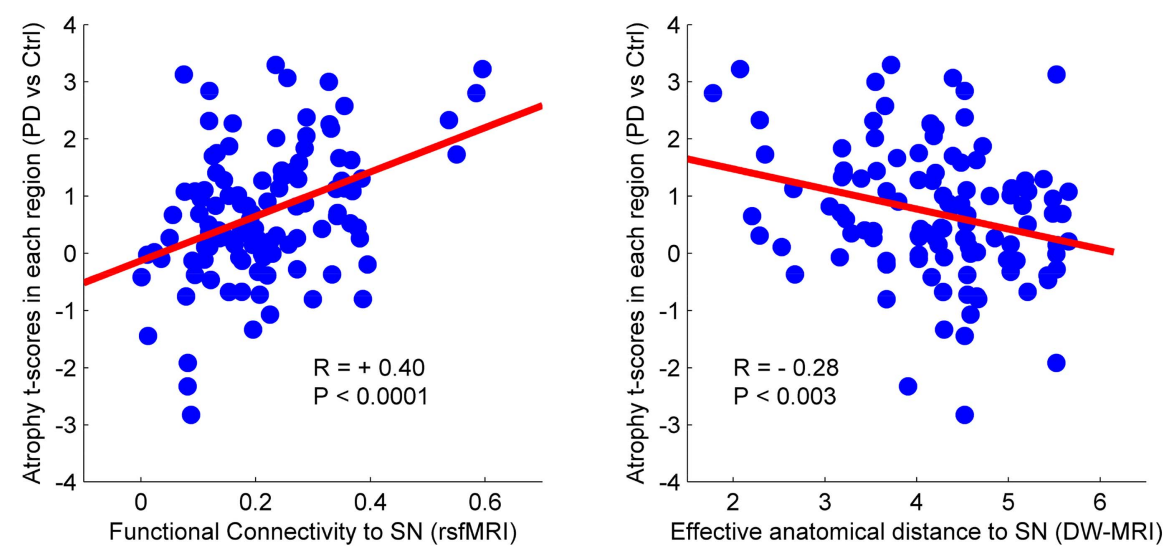

Figure 4. Relationship between atrophy in different brain regions and functional and structural connectivity with SN. The brain was parcellated into 112 regions (Figure 4-figure supplement 1). SN was chosen a priori as the region of interest, and the functional and structural connectivities between each given region and SN were calculated. The statistical difference ( $\mathrm{t}$-score) between the average deformation in PD and controls in each region was used as an atrophy measure. Using correlation, the relationship between regional atrophy and both regional functional connectivity with SN using resting-state fMRI (rsfMRI) (left) and regional anatomical distance using diffusionweighted imaging (DW-MRI) (right) was examined. There was significantly greater atrophy with proximity to the SN determined functionally $(r=0.4, p<0.0001)$ and anatomically $(r=-0.28, p<0.003)$. Note that the connectivity measure in rsfMRI is correlation, resulting in greater values for more connected regions, whereas the connectivity measure in DW-MRI is distance, resulting in smaller values for more connected regions.

DOI: 10.7554/eLife.08440.015

The following source data and figure supplement are available for figure 4:

Source data 1. Atlas labels and their anatomical coordinates.

DOI: 10.7554/eLife.08440.016

Source data 2. Best propagators (DW-MRI connectome).

DOI: 10.7554/eLife.08440.017

Figure supplement 1. Anatomical atlas used for regional analysis.

DOI: 10.7554/eLife.08440.018 
Table 3. Best propagators (resting-state fMRI connectome)

\begin{tabular}{|c|c|c|c|}
\hline Seed region & $\mathbf{r}$ & Seed region & $\mathbf{r}$ \\
\hline Substantia nigra & 0.40 & Cerebellum VIIIb & 0.05 \\
\hline Subthalamic nucleus & 0.28 & Insula & 0.04 \\
\hline Red nucleus & 0.28 & Anterior temporal lobe (lateral part) & 0.02 \\
\hline Cerebellum dentate & 0.27 & Cerebellum Crusl & 0.01 \\
\hline Pallidum & 0.23 & Superior temporal gyrus (anterior part) & 0.01 \\
\hline Hippocampus & 0.22 & Caudate nucleus & -0.06 \\
\hline Cerebellum vermis $X$ & 0.21 & Superior temporal gyrus (posterior part) & -0.07 \\
\hline Cerebellum vermis VIIla & 0.20 & Middle and inferior temporal gyrus & -0.07 \\
\hline Cerebellum interposed & 0.20 & Lingual gyrus & -0.08 \\
\hline Cerebellum fastigial & 0.20 & Postcentral gyrus & -0.08 \\
\hline Cerebellum vermis IX & 0.20 & Precentral gyrus & -0.09 \\
\hline Cerebellum vermis VIIIb & 0.18 & Posterior temporal lobe & -0.09 \\
\hline Cerebellum I IV & 0.18 & Inferior frontal gyrus & -0.10 \\
\hline Cerebellum vermis VIIb & 0.17 & Middle frontal gyrus & -0.10 \\
\hline Parahippocampal gyrus & 0.16 & Cuneus & -0.10 \\
\hline Cerebellum V & 0.16 & Anterior cingulate gyrus & -0.12 \\
\hline Anterior temporal lobe (medial part) & 0.15 & Occipital lobe (lateral part) & -0.12 \\
\hline Cerebellum vermis Crusll & 0.14 & Lateral orbital gyrus & -0.16 \\
\hline Occipitotemporal gyrus (lateral part) & 0.14 & Superior frontal gyrus & -0.16 \\
\hline Cerebellum VIlb & 0.13 & Parietal lobe (Inferiolateral) & -0.16 \\
\hline Cerebellum Crusll & 0.13 & Superior parietal gyrus & -0.20 \\
\hline Cerebellum IX & 0.12 & Pre-subgenual frontal cortex & -0.20 \\
\hline Cerebellum VI & 0.12 & Posterior orbital gyrus & -0.23 \\
\hline Amygdala & 0.12 & Posterior cingulate gyrus & -0.23 \\
\hline Cerebellum X & 0.11 & Medial orbital gyrus & -0.27 \\
\hline Cerebellum vermis Crusl & 0.10 & Straight gyrus & -0.31 \\
\hline Putamen & 0.10 & Anterior orbital gyrus & -0.33 \\
\hline Cerebellum vermis $\mathrm{VI}$ & 0.08 & Subgenual frontal cortex & -0.34 \\
\hline Cerebellum VIIla & 0.07 & Nucleus accumbens & -0.38 \\
\hline Thalamus & 0.06 & Subcallosal area & -0.42 \\
\hline
\end{tabular}

Each brain region from the atlas was used as a potential propagator. The statistical difference (t-value) between the average deformation in PD and controls in each region was used as an atrophy measure. The correlation between this atrophy measure and the functional connectivity to the potential propagator was used as a measure of propagation strength. The potential propagator regions are sorted by correlation values.

fMRI: functional MRI, PD: Parkinson's disease.

DOI: 10.7554/eLife.08440.019

volumes of brainstem, basal ganglia, and cerebral cortex. In the SN, measurements of volume and shape have been inconclusive, reporting either no change, a decrease, or increase in volume depending on the method used (Pyatigorskaya et al., 2014). However, most studies using parametric mapping have failed to report a difference in SN. Reduced putamen or caudate volume has been reported but only in advanced cases with mild cognitive impairment or dementia (Apostolova et al., 2010; Silbert and Kaye, 2010; Pyatigorskaya et al., 2014). Similarly, hippocampus and amygdala atrophy are typically linked to cognitive impairment (for review see Silbert and Kaye, 2010; Ibarretxe-Bilbao et al., 2011). With regard to cortical areas, studies using voxel-based morphometry (VBM), DBM, or cortical thickness measurements have reported significant differences between PD and controls in parahippocampal gyrus, inferior, middle and frontal temporal 
Table 4. Clinical characteristics of subjects

\begin{tabular}{|c|c|c|c|}
\hline & Control $(n=117)$ & $P D(n=232)$ & p value \\
\hline Age (years) & $59.7 \pm 11.3$ & $61.2 \pm 9.1$ & 0.1 \\
\hline Years of education (years) & $15.7 \pm 2.91$ & $15.4 \pm 2.8$ & NS \\
\hline Sex (M/F/\% males) & $74 / 43 / 63.2$ & $155 / 77 / 66.8$ & NS \\
\hline Handedness-R/L/A & $98 / 11 / 8$ & $210 / 17 / 5$ & NS \\
\hline Striatum binding ratio & $2.6 \pm 0.6$ & $1.4 \pm 0.4$ & $<0.0001$ \\
\hline MoCA & $28.2 \pm 1.2$ & $27.3 \pm 2.2$ & $<0.0001$ \\
\hline Disease duration (months) & - & $6.9 \pm 7.1$ & - \\
\hline MDS UPDRS part III & - & $21.9 \pm 9.1$ & - \\
\hline H\&Y stage & - & $1.6 \pm 0.5$ & - \\
\hline \multicolumn{4}{|c|}{$\begin{array}{l}\text { M = male, F = female, NS = not significant, H\&Y: Hoehn and Yahr, PD: Parkinson's disease, MoCA, Montreal } \\
\text { Cognitive Assessment, UPDRS, Unified Parkinson's Disease Rating Scale. Statistical differences analyzed through an } \\
\text { unpaired t-test or chi square test. Listed values are the mean } \pm \text { standard deviation. } \\
\text { DOI: } 10.7554 / \text { Life. } 08440.020\end{array}$} \\
\hline
\end{tabular}

gyrus, parietal lobe and occipital lobe, but, once again, typically in advanced patients with cognitive impairment (Silbert and Kaye, 2010; Ibarretxe-Bilbao et al., 2011; Camicioli, 2013; Pyatigorskaya et al., 2014). Our finding of an atrophy network including brainstem and subcortical regions in these de novo patients is likely due to the larger number of participants available through the PPMI database compared to previous relatively underpowered investigations.

Eidelberg and colleagues (Eidelberg, 2009) were the first to use data reduction techniques (principal component analysis) to map PD pathology using $\left[{ }^{18} \mathrm{~F}\right]$ fluorodeoxyglucose (FDG) positron emission tomography. They identified a PD-related pattern (PDRP) implicating several regions identified in our analysis, namely globus pallidus, putamen, thalamus, premotor and supplementary motor areas. The expression of this PDRP differentiates patients from healthy controls and correlates with measures of disease severity. However, it probably reflects functional effects of the disease more than neuronal atrophy, as evidenced by the fact that it is normalized by levodopa or deep brain stimulation (Eidelberg, 2009).

\section{PD targets an intrinsic brain network}

We found that the set of regions demonstrating atrophy in the PD group corresponded spatially to one ICN in normal brain. rsfMRI and anatomical imaging have identified consistent sets of regions that act as functional networks, by virtue of anatomical connectivity and temporal covariance of neuronal activity. We showed that our PD-ICA set of regions corresponded to a normal ICN identified by seedbased resting-state functional connectivity and ICA (Smith et al., 2009, 2013). The finding that PD targets a set of connected brain regions supports the network-spread hypothesis, although an alternate explanation is that neurons in one network could share a common vulnerability. The brain regions that make up the PD-ICA network are involved in reward and motivation, as demonstrated by the fact that they respond to the subjective value of a perceived stimulus during fMRI (Bartra et al., 2013). All the regions of this value network receive projections from midbrain dopamine neurons, in keeping with this neurotransmitter's role in signaling incentive value (Salamone et alı, 2005; Berridge, 2006).

\section{Network spread in PD}

Recent evidence supports a hypothesis originally put forward by Braak (Braak et al., 2003, 2006; Goedert et al., 2013), according to which pathogenic forms of the protein alpha-synuclein spread throughout the nervous system leading to a stereotypical step-wise pattern of neurological impairment in PD. Misfolded synuclein fibrils injected focally into rodent brains spread to neuronally connected but not adjacent areas (Luk et al., 2012; Masuda-Suzukake et al., 2013) (see also Figure 1-figure supplement 3).

Here, we tried to identify a putative best propagator or epicenter of the disease by creating functional and anatomical connectomes from an anatomical brain atlas. We showed that the SN could 
act as a propagator, since the rate of atrophy in any brain region was proportional to its effective topological distance from the SN, as determined by either functional (rsfMRI) or anatomical (DW-MRI) connectivity. We note that, while the SN contains dopamine neurons whose degeneration accounts for all the key clinical features of early PD, the Braak scheme identifies the dorsal motor nucleus (DMN) of the vagus in the medulla as the first affected CNS structure. Indeed, recent evidence supports the potential transfer of pathogenic alpha-synuclein from the intestine to the DMN via the vagus nerve (Holmqvist et al., 2014). In the current study, the DMN did not demonstrate atrophy in PD compared to control subjects. Perhaps this is due to insensitivity of T1-weighted MRI-based anatomical methods in identifying neuronal loss in lower brainstem structures. The DMN is much smaller that the SN (approximately 1/10-1/20 in volume) and has limited contrast in T1 MRI. Our results, however, suggest that the SN could act as the propagator of the disease to the supratentorial CNS. Note, however, that spatial resolution limitations for all of the imaging modalities used here, T1-weighted MRI, fMRI, and DW-MRI, make it difficult to localize atrophy to small nuclei with complete certainty. Spatial inaccuracy in atlas generation and normalization of subject images to Montreal Neurological Institute (MNI) space may compound this problem. This is especially relevant to small structures of the basal forebrain and midbrain. For example, the propagator analysis identified SN, subthalamic nucleus, and red nucleus as potential propagators, but it is clear that the imaging techniques used here do not allow us to fully resolve these structures, either anatomically or functionally. Furthermore, because T1-weighted MRI is sensitive to iron content, changes in iron accumulation, for example, in SN, globus pallidus, or red nucleus, may be interpreted as volume changes by the DBM methodology.

Nonetheless, comparing the atrophy pattern identified here to the stages described by Braak et al. (2003), Braak et al. (2006), we note that all the areas identified in stage 3 (pedunculopontine nucleus, amygdala, basal forebrain, and substantia nigra), stage 4 (temporal mesocortex and hippocampus), and stage 5 (insula, cingulate cortex, and temporal neocortex) belong to our PD-ICA network. Braak hypothesized that the medial temporal lobe served as a beachhead for further propagation to the remaining cortex, and interestingly three of the best supratentorial propagators identified here were the parahippocampal gyrus, anterior medial temporal lobe, and hippocampus (Table 3).

Another prediction of the network-spread model is that connectome hubs should be especially vulnerable to disease spread (Zhou et al., 2012). Hubs are defined as brain regions that are highly connected using graph theoretical metrics such as degree (Crossley et al., 2014) or betweenness (He et al., 2009). In theory, hubs, being highly connected, should have greater exposure to a toxic agent that is spread trans-neuronally. In a recent meta-analysis, Crossley et al. (2014) found that most neurodegenerative diseases, including $A D$ and fronto-temporal dementia, did indeed target hubs. However, one salient exception was PD. One possibility is that the PD studies included in the metaanalysis were too small in scale or methodologically incapable of detecting the true extent of damage. It is intriguing that our study identified numerous hub regions (Bassett et al., 2008; He et al., 2009; Crossley et al., 2014) in the PD-ICA network, including the medial temporal lobe, putamen, insula, occipital cortex, anterior cingulate, superior frontal gyrus, and middle frontal gyrus. One notable hub region absent from our PD-ICA network is the posterior cingulate gyrus/precuneus, an area typically affected in AD (Buckner et al., 2009). It would be interesting to see if this area eventually develops atrophy as PD progresses, and whether this is associated with cognitive impairment.

\section{Atrophy in healthy aged subjects}

The PD-ICA network demonstrated a correlation between atrophy and dopamine denervation measured with SPECT, in both PD patients and age-matched control subjects. Atrophy and dopamine denervation also both correlated with age in the control group. In sum, the control subjects demonstrated age-related loss of dopamine innervation and atrophy in the PD-ICA network. This finding is also novel. It is known that healthy aging is associated with a progressive loss of dopamine neurotransmission (Fearnley and Lees, 1991), and that this may account for motor slowing and executive cognitive impairment that occurs with age (Jagust, 2013). Indeed, subtle Parkinsonian signs such as stooped posture, bradykinesia, and reduced facial expression in the healthy elderly were associated with SN neuron loss at post-mortem (Ross et al., 2004). Our results extend these findings by showing that neurodegeneration of the extended dopamine network also occurs in healthy aging, albeit without attaining the severity of outright PD, possibly via loss of neurotrophism, or perhaps, due 
to low-level mitochondrial dysfunction or synucleinopathy (Olanow and Brundin, 2013). PD might then target the PD-ICA network due to a dual hit effect of pathology superimposed upon normal aging.

\section{Materials and methods}

\section{Subjects and data collection}

Data used in this article were primarily obtained from the PPMI database. In addition, rsfMRI and DWMRI in healthy subjects were used to generate normal human connectomes for investigation of the network propagation model.

\section{PPMI data set}

The PPMI is described at www.ppmi-info.org. PPMI is a public-private partnership funded by the Michael J Fox Foundation for Parkinson's Research and funding partners listed at www.ppmi-info.org/ fundingpartners. It is an observational, multicenter longitudinal study designed to identify PD biomarkers (Marek et al., 2011). Each participating PPMI site received approval from a local research ethics committee before study initiation and obtained written informed consent from all subjects participating in the study.

For this study, we used the initial visit 3T high-resolution T1-weighted MRI scans acquired from September 2013 to January 2014. MRI data were acquired in 16 centers participating in the PPMI project, using scanners from three different manufacturers (GE medical systems, Siemens, and Philips medical systems). The acquisition parameters are detailed in the data set Website: http://www.ppmiinfo.org/wp-content/uploads/2015/03/PPMI-MRI-Operations-Manual-V7-0-20JAN2015-FINAL.pdf.

We also made use of the following data for each participant: age, sex, disease duration, score on the Movement Disorder Society_UPDRS III (Goetz et al., 2008) while off medications, score on the Montreal Cognitive Assessment battery and striatal binding ratio (SBR) from SPECT measurements with the tracer $\left[{ }^{123}\right.$ I]FP-CIT, a measure of dopamine neuron terminal density (Booij and Knol, 2007). In total data from 355 subjects (237 PD patients and 118 age-matched controls) were used. Six subjects, five PD patients and one age-matched control, were excluded from analysis due to failure in MRI processing. Clinical characteristics are shown in Table 4.

\section{rsfMRI}

We acquired rsfMRI in 51 healthy, right-handed volunteers (mean age: $23.6 \pm 5.9$, range: 18-47, 32 men, 63\%). None of the subjects reported a history of drug abuse, neurological or psychiatric disorder. The experimental protocol was reviewed and approved by Research Ethics Board of MNI. All subjects gave informed consent. Scans were acquired using a Siemens MAGNETOM Trio 3T MRI system at the MNI. High-resolution, T1-weighted, three-dimensional volume acquisition for anatomic localization (1- $\mathrm{mm}^{3}$ voxel size) and resting-state echoplanar T2*-weighted images with blood oxygenation level-dependent (BOLD) contrast (3.5-mm isotropic voxels, TE $30 \mathrm{~ms}$, TR $2 \mathrm{~s}$, flip angle $90^{\circ}$ ) were acquired from all participants. Each resting-state scan was 5-min long (150 vol). Subjects were instructed to rest quietly with eyes open.

\section{DW-MRI}

To obtain white matter connectivity maps of normal brain, we used the Illinois Institute of Technology Human Brain Atlas v.3 (Varentsova et al., 2014) (http://www.nitrc.org/projects/iit2/). This atlas contains structural (T1) and high angular resolution DW-MRI data, and probabilistic gray matter maps of the adult human brain in MNI space. The atlas was generated from MRI data from 72 human subjects (42 females (59\%): mean age $26.6 \pm 4.8$ years, range $20-39$ years; 30 males: mean age $31.9 \pm$ 4.9 years, range $22-40$ years).

\section{DBM}

Local change in tissue density was calculated using DBM. DBM consists in spatially transforming each MRI non-linearly to a stereotaxic template, and using the local deformation as a measure of tissue expansion or atrophy. There are other methods to detect population differences in brain structure. VBM measures local gray matter density by transforming the brain to stereotaxic space, segmenting 
the tissue into gray and white matter and cerebrospinal fluid, and performing spatial smoothing on the gray matter maps so that local image intensity reflects gray matter density (Ashburner and Friston, 2000). We decided against VBM as it does not preserve the entirety of the MRI data, and there is some suggestion that it is less sensitive than DBM to subcortical atrophy (Borghammer et al., 2010; Scanlon et al., 2011). Another approach is to measure cortical thickness from the MRI (Pereira et al., 2014), but this would also make it impossible to detect subcortical changes.

For DBM, we registered every brain non-linearly to the MNI152-2009c template (available at http:// www.bic.mni.mcgill.ca/ServicesAtlases/ICBM152NLin2009) and computed the deformation applied at each voxel using the procedure explained in Aubert-Broche et al. (2013). Pre-processing of MRI included denoising using optimized non-local means filtering (Coupe et al., 2008), correction for intensity inhomogeneity (Sled et al., 1998), and linear intensity scaling using histogram matching to the MNI152-2009c template.

The resulting images were registered to $\mathrm{MNI}$ space using the MNI152-2009c template, in two steps: (1) A hierarchical nine-parameter linear registration was computed between native MRI images and the template by maximizing the cross correlation of intensities as the similarity measure (Collins et al., 1994). The resulting transformation was applied to the MR image to resample it onto a $1-\mathrm{mm}^{3}$ voxel grid and bring it into $\mathrm{MNI}$ space. (2) A hierarchical non-linear registration was performed on the linearly resampled scan to align it with the MNI152-2009c template (Collins and Evans, 1997). The resulting non-linear transformation field was inverted to generate a map of the deformations in template (MNI) space for each subject. Quality control was performed on each individual data set: the brain mask, and linear and nonlinear registrations were visually inspected, and data sets with faulty registration were discarded.

After the registration procedure, for each MRI and for each position in the brain $x=x 1, x 2, x 3$, we obtain a displacement value in each direction to generate a field of vectors: $U(x)=(u 1(x), u 2(x), u 3(x))$; that is, during the registration procedure position, $x$ is displaced to the new position $x+U(x)$ in the template space. This shows how much each voxel was moved from the MNI152-2009c template to match the subject's brain. To estimate local atrophy, an extra step is needed. Since a completely uniform displacement results in no change of volume, we are interested in the derivative of the displacement in each direction, that is, the determinant of the local Jacobian matrix of displacement.

$$
J=\frac{\partial U}{\partial x}=\left[\begin{array}{lll}
\frac{\partial u_{1}}{\partial x_{1}} & \frac{\partial u_{1}}{\partial x_{2}} & \frac{\partial u_{1}}{\partial x_{3}} \\
\frac{\partial u_{2}}{\partial x_{1}} & \frac{\partial u_{2}}{\partial x_{2}} & \frac{\partial u_{2}}{\partial x_{3}} \\
\frac{\partial u_{3}}{\partial x_{1}} & \frac{\partial u_{3}}{\partial x_{2}} & \frac{\partial u_{3}}{\partial x_{3}}
\end{array}\right]
$$

This Jacobian matrix is estimated using a first order approximation:

$$
\frac{\partial u_{3}}{\partial x_{2}} \cong \frac{u_{3}\left(x_{1}, x_{2}+\delta, x_{3}\right)-u_{3}\left(x_{1}, x_{2}-\delta, x_{3}\right)}{2 \cdot \delta},
$$

where $\delta$ is the voxel dimension along the $x_{2}$ axis. To calculate the relative ratio of the local volume change, we use the determinant of the Jacobian matrix $|\mathrm{J}|$ minus 1 , that is, $|\mathrm{J}|-1$. By performing this calculation at each voxel, we obtain a map of local relative volumetric difference between each subject image and the MNI152-2009c template, reported in the MNI152-2009c template Talairach-like coordinate system (Chung et al., 2001).

\section{Anatomical atlas}

We created a composite anatomical atlas of gray matter regions in the cerebral hemispheres, cerebellum, and midbrain. For the supratentorial regions, we used the Hammers atlas (Copyright Imperial College of Science, Technology and Medicine, Alexander Hammers and University College London 2011. All rights Reserved) (Hammers et al., 2003) excluding the brainstem, cerebellum, and white matter. For the cerebellum, we used a public-domain high-resolution digital cerebellar atlas (Diedrichsen et al., 2009). These two atlases do not have adequate segmentation of three midbrain structures, the SN, subthalamic nucleus, and red nucleus. We therefore manually segmented these three structures using the Display software tool (McConnell Brain Imaging Centre) and three sources 
of anatomical information: the high-resolution MRI template (T1-weighted ICBM 2009c template, resolution $=0.5 \mathrm{~mm}^{3}$ ), the BigBrain (Amunts et al., 2013), a 20-micron resolution digital brain atlas in MNI space, and the brainstem anatomical atlas of Duvernoy et al. (1995). The three structures were manually drawn on the high-resolution ICBM 2009c template. We then confirmed the segmentation of these three regions with a recently developed subcortical atlas based on ultra high-field MRI (Keuken et al., 2014). The composite atlas thus created contains 112 cortical and subcortical structures (Figure 4-figure supplement 1, Figure 4-source data 1) and excludes all brainstem regions caudal to the SN.

\section{Extracting independent components of the structural deformation}

We used ICA to extract patterns of deformation in PD patients and age-matched controls. ICA is a statistical method to decompose multivariate data into statistically independent components (Calhoun et al., 2001; Beckmann and Smith, 2004; Hyvärinen et al., 2004). In this case, we used ICA to decompose the deformation maps into spatially distinct subcomponents. ICA was performed with MELODIC (http://fsl.fmrib.ox.ac.uk/fsl/fslwiki/MELODIC), a toolbox that is part of the FSL analysis package (Beckmann and Smith, 2004, 2005; Smith et al., 2004; Douaud et al., 2014). All the individual DBM images (patients and controls combined) were concatenated to create a 4-D image in which the first three dimensions are the individual 3-D deformation maps and the fourth dimension consists of the subjects. MELODIC applies an initial principal component analysis-based dimension estimation to find the optimal number of independent components and then uses this number in the decomposition procedure to identify the independent spatial components. Each of these components consists of a vector of normalized DBM values (one per subject) as well as a corresponding 3-D spatial map. The spatial maps were converted to z-statistic images via normalized mixture-model fitting, and thresholded at $z=3$ (Beckmann and Smith, 2004; Smith et al., 2009). The ICA algorithm in MELODIC is sensitive to sparsely distributed (super-Gaussian) data (Daubechies et al., 2009) as typically seen in fMRI. We confirmed that the DBM data used here possessed this super-Gaussian property (kurtosis $>4$ ).

\section{Statistical analysis of independent networks}

To identify regions showing greater atrophy in PD, the average DBM values for each ICA component and each subject were entered in an unpaired t-test comparing PD subjects and age-matched controls (Bonferroni corrected for multiple comparisons). Also, the DBM values from each component were correlated with the age of each subject to identify patterns of deformation associated with aging. Again, Bonferroni corrections were applied. The DBM values from the component(s) that demonstrated a statistically significant group difference were compared to two disease-related clinical measures using linear regression: SBR and UPDRS III.

\section{rsfMRI analysis}

In order to test the theory that PD targets normal brain networks, we analyzed rsfMRI data from 51 young healthy individuals. The rsfMRI data were preprocessed using the Neuroimaging Analysis Kit (NIAK) (Bellec et al., 2010, 2012), to perform slice timing correction, rigid body motion correction, and removal of slow temporal drift using a high-pass filter with $0.01 \mathrm{~Hz}$ cut-off (Perlbarg et al., 2007). Physiological noise was accounted for by including white matter and cerebrospinal fluid signals as covariates. In the next step, the mean motion-corrected volume of each subject's fMRI data was first linearly (6 parameters: 3 translations, 3 rotations) registered to the native individual T1 image and then non-linearly registered to the MNI152 non-linear template (http://www.bic.mni.mcgill.ca/ServicesAtlases/HomePage). All data were resampled and smoothed with a 6-mm Gaussian kernel. All fMRI time series further underwent level one auto-regression (AR1) temporal de-noising.

The mask of the SN described above was used to extract the average BOLD time series of each individual scan. The time series from right and left SN were averaged. Seed-based functional connectivity analysis was performed (Worsley et al., 2002; Worsley, 2005) as implemented in fmristat (http://www.math.mcgill.ca/keith/fmristat/). The SN average BOLD signal was entered as a regressor in the design matrix and its correlation calculated with all voxels in the brain. A mixed effects model was applied to generate a t-map for the group. This was thresholded based on random field theory to achieve a $\mathrm{p}$ value of 0.05 after correction for multiple comparisons. 


\section{Comparing PD-related structural atrophy networks and functional networks in healthy brain}

We quantified the spatial similarity between PD-ICA atrophy maps and intrinsic networks in the healthy brain. We compared the PD-ICA map to intrinsic brain networks obtained from three different sources: the functional connectivity map obtained from the SN seed region, and ICNs from two published sources (Smith et alo, 2009, 2013). We also compared the PD-ICA network map to a map derived from a metaanalysis of fMRI experiments where subjects tracked the value of rewarding stimuli (Bartra et al., 2013).

First, the functional connectivity map obtained from the $\mathrm{SN}$ seed-based analysis was compared to the 30 different ICA maps from the deformation analysis. Second, we compared the PD atrophy map (PD-ICA network) to the 70 ICNs identified by Smith et al. based on ICA analysis of resting-state data in healthy subjects (Smith et al., 2009). We further compared the PD-ICA map to 100 ICNs identified from the HCP rsfMRI data (Smith et al., 2013). Then, we compared the 30 ICA maps from the deformation analysis to a map identified in a meta-analysis of value-related fMRI studies, reasoning that dopamine networks implicated in PD would be similar to value networks in healthy brains (i.e., regions where BOLD signal tracks the value of experimental stimuli). Spatial cross-correlation (Pearson) was used as the measure of similarity. We chose $|r|>0.25$ as indicative of similarity between two spatial maps as this has been argued to guard against false positives in a similar comparison between ICA-derived spatial maps (Smith et al., 2009).

\section{Relating deformation to the normal connectome}

We generated the normal connectome of our 112 region brain atlas (52 paired bilateral regions, 8 midline regions), in two different ways, with rsfMRI and with DW-MRI. The goal here was to test the theory that geodesic (synaptic) proximity to the SN in healthy brain would predict the distribution of regional atrophy in PD.

\section{Generation of the functional connectome}

The modified Hammers-Cerebellum-Brainstem atlas was used to extract the average BOLD timeseries, after correction for physiological noise, for all regions for each of the $51 \mathrm{rsfMRI}$ acquisitions. All 112 regions in the atlas were used as separate masks and average time series were extracted for each. The time series used were the residuals of the linear model after correction for physiological noise and head motion. As a result, we obtained the correlation between all region pairs, which gives a $112 \times$ 112 connectivity matrix for each acquisition. Then, a common group-based matrix called $R_{\text {group }}$ was calculated. To do so, we followed steps explained in Carbonell et al. (2014): each individual subject's (j) correlation matrix $R_{\text {ind }}^{j}$ was converted to a standard normal metric using the Fisher transformation

$$
Z_{\text {ind }}^{j}=\frac{1}{2} \log \left(\frac{1+R_{\text {ind }}^{j}}{1-R_{\text {ind }}^{j}}\right) .
$$

Then, all the Fisher transformed results were averaged.

$$
Z_{\text {group }}=\frac{\sum_{j=1}^{N} Z_{\text {ind }}^{j}}{N},
$$

where $N$ is the number of subjects. Finally, the group $Z$ correlation matrix result was converted back to the correlation space using an inverse Fisher transform

$$
R_{\text {group }}=\frac{e^{2 Z_{\text {group }}}-1}{e^{2 Z_{\text {group }}}+1}
$$

The modified Hammers-Suit-Brainstem atlas was also used to extract the average deformation values (Jacobian) for each region for each PD and age-matched control subject. A t-test was performed in each region to assess the difference between the PD and control groups, corrected for age. These $t$-values are a measure of the difference between the two groups and the sign of the t-value shows the direction of the effect, with negative values chosen to mean greater deformation (atrophy) in PD. 
The relationship between functional connectivity of each brain region with the selected seed region (SN) and the t-value of the deformation in each region (PD minus age-matched controls) was investigated using correlation analysis.

\section{Generation of the DW-MRI connectome}

Probabilistic anatomical connectivity values between each pair of atlas regions were estimated using a fully automated fiber tractography algorithm (Iturria-Medina et al., 2007) and the intravoxel fiber orientation distribution functions from the IIT Human Brain Atlas v.3 (Varentsova et al., 2014). A maximum of $500-\mathrm{mm}$ trace length and a curvature threshold of $\pm 90^{\circ}$ were imposed as tracking parameters. Based on the resulting voxel-region connectivity maps, the anatomical connection probability $(A C P)$ between any pair of regions $i$ and $j\left(A C P_{i j}=A C P_{j i}\right)$ was calculated as the maximum voxel-region connectivity value between both regions. The ACP measure (Iturria-Medina et al., 2007) reflects the degree of evidence supporting the existence of each hypothetical white matter connection, independently of the density/strength of this connection, and is thus a measure of low susceptibility to gross fiber degeneration related to the aging processes. Then, effective anatomical distances between each region $\mathrm{i}$ and all the other $\mathrm{RO}$ were estimated as the lengths of the shortest paths (in terms of ACP values) linking that region to all the other regions (Iturria-Medina et al., 2014).

Then, as for the functionally derived connectome above, we tested whether the PD minus control atrophy value in each atlas region depended on the geodesic distance to the SN in the DW-MRI connectome. We calculated the non-linear correlation between the regional $T$ value of the difference in Jacobian between patients and controls and the effective anatomical distance to the SN.

\section{Acknowledgements}

This work was funded by grants from the Michael J Fox Foundation for Parkinson's Research, the W Garfield Weston Foundation, and the Alzheimer's Association, the Canadian Institutes for Health Research, and the Natural Sciences and Engineering Research Council of Canada. We thank Christian Beckmann and Simon Eickhoff for their advice on data analysis. Data used in this article were obtained from the Parkinsons Progression Markers Initiative (PPMI) database (www.ppmi-info.org/data). For up-to-date information on the study, visit www.ppmi-info.org. PPMl is sponsored and partially funded by the Michael J Fox Foundation for Parkinsons Research and funding partners, including AbbVie, Avid Radiopharmaceuticals, Biogen, Bristol-Myers Squibb, Covance, GE Healthcare, Genentech, GlaxoSmithKline (GSK), Eli Lilly and Company, Lundbeck, Merck, Meso Scale Discovery (MSD), Pfizer, Piramal Imaging, Roche, Servier, and UCB (www.ppmi-info.org/fundingpartners). Some data used in this paper were also provided by the Human Connectome Project, WU-Minn Consortium (Principal Investigators: David Van Essen and Kamil Ugurbil; 1U54MH091657) funded by the $16 \mathrm{NIH}$ Institutes and Centers that support the NIH Blueprint for Neuroscience Research; and by the McDonnell Center for Systems Neuroscience at Washington University.

\section{Additional information}

Funding

\begin{tabular}{|c|c|c|}
\hline Funder & Grant reference & Author \\
\hline $\begin{array}{l}\text { Michael J. Fox Foundation for } \\
\text { Parkinson's Research (MJFF) }\end{array}$ & 320897 & D Louis Collins, Alain Dagher \\
\hline W. Garfield Weston Foundation & 320897 & D Louis Collins, Alain Dagher \\
\hline Alzheimer's Association & 320897 & D Louis Collins, Alain Dagher \\
\hline $\begin{array}{l}\text { Canadian Institutes of Health } \\
\text { Research (Instituts de recherche } \\
\text { en santé du Canada) }\end{array}$ & MOP-136776 & Alain Dagher \\
\hline $\begin{array}{l}\text { Natural Sciences and } \\
\text { Engineering Research Council of } \\
\text { Canada (Conseil de Recherches } \\
\text { en Sciences Naturelles et en } \\
\text { Génie du Canada) }\end{array}$ & $436259-13$ & Alain Dagher \\
\hline
\end{tabular}

The funders had no role in study design, data collection and interpretation, or the decision to submit the work for publication. 
Author contributions

YZ, MU, DLC, Conception and design, Analysis and interpretation of data, Drafting or revising the article; YI-M, MD, YZ, KM-HL, VF, ACE, Analysis and interpretation of data, Drafting or revising the article; AD, Conception and design, Acquisition of data, Analysis and interpretation of data, Drafting or revising the article

Author ORCIDs

Alain Dagher, (iD http://orcid.org/0000-0002-0945-5779

Ethics

Human subjects: For the Parkinson's Progression Markers Initiative (PPMI) database (www.ppmi-info. org/data). Each participating PPMI site received approval from a local research ethics committee before study initiation, and obtained written informed consent from all subjects participating in the study. For the resting state fMRI data collected in our lab, We acquired resting state fMRI in 51 healthy, right-handed volunteers. The experimental protocol was reviewed and approved by Research Ethics Board of Montreal Neurological Institute. All subjects gave informed consent.

\section{Additional files}

Major datasets

The following dataset was generated:

\begin{tabular}{|c|c|c|c|c|}
\hline Author(s) & Year & Dataset title & $\begin{array}{l}\text { Dataset ID } \\
\text { and/or URL }\end{array}$ & $\begin{array}{l}\text { Database, license, and } \\
\text { accessibility information }\end{array}$ \\
\hline $\begin{array}{l}\text { Zeighami Y, Ulla M, } \\
\text { Iturria-Medina Y, } \\
\text { Dadar M, Zhang Y, } \\
\text { Larcher KM-HL, Fonov V, } \\
\text { Evans AC, Collins DL, } \\
\text { Dagher A }\end{array}$ & 2015 & $\begin{array}{l}\text { Network structure of } \\
\text { brain atrophy in de novo } \\
\text { Parkinson's Disease }\end{array}$ & $\begin{array}{l}\text { http://neurovault.org/ } \\
\text { collections/860/ }\end{array}$ & $\begin{array}{l}\text { Publicly available at } \\
\text { NeuroVault } \\
\text { (collection 860). }\end{array}$ \\
\hline
\end{tabular}

The following previously published datasets were used:

\begin{tabular}{|c|c|c|c|c|}
\hline Author(s) & Year & Dataset title & $\begin{array}{l}\text { Dataset ID } \\
\text { and/or URL }\end{array}$ & $\begin{array}{l}\text { Database, license, and } \\
\text { accessibility information }\end{array}$ \\
\hline Marek K et al, & 2011 & $\begin{array}{l}\text { Parkinson Progression } \\
\text { Marker Initiative (PPMI) }\end{array}$ & $\begin{array}{l}\text { http://www.ppmi- } \\
\text { info.org/ }\end{array}$ & $\begin{array}{l}\text { PPMI data and specimens } \\
\text { are made accessible } \\
\text { through the Web site to } \\
\text { academic and industry } \\
\text { researchers to perform } \\
\text { verification studies of PD } \\
\text { biomarkers. Investigators } \\
\text { are required to submit } \\
\text { basic information about } \\
\text { themselves for basic } \\
\text { administrative review to } \\
\text { ensure legitimacy. Upon } \\
\text { approval, investigators will } \\
\text { be given immediate access. }\end{array}$ \\
\hline
\end{tabular}

\section{References}

Amunts K, Lepage C, Borgeat L, Mohlberg H, Dickscheid T, Rousseau MÉ, Bludau S, Bazin PL, Lewis LB, OrosPeusquens AM, Shah NJ, Lippert T, Zilles K, Evans AC. 2013. BigBrain: an ultrahigh-resolution 3D human brain model. Science 340:1472-1475. doi: 10.1126/science.1235381.

Apostolova LG, Beyer M, Green AE, Hwang KS, Morra JH, Chou YY, Avedissian C, Aarsland D, Janvin CC, Larsen JP, Cummings JL, Thompson PM. 2010. Hippocampal, caudate, and ventricular changes in Parkinson's disease with and without dementia. Movement Disorders 25:687-695. doi: 10.1002/mds.22799.

Ashburner J, Friston KJ. 2000. Voxel-based morphometry-the methods. Neuroimage 11:805-821. doi: 10.1006/ nimg.2000.0582.

Aubert-Broche B, Fonov VS, García-Lorenzo D, Mouiha A, Guizard N, Coupé P, Eskildsen SF, Collins DL. 2013. A new method for structural volume analysis of longitudinal brain MRI data and its application in studying the growth trajectories of anatomical brain structures in childhood. Neuroimage 82:393-402. doi: 10.1016/j. neuroimage.2013.05.065.

Bartra O, McGuire JT, Kable JW. 2013. The valuation system: a coordinate-based meta-analysis of BOLD fMRI experiments examining neural correlates of subjective value. Neuroimage 76:412-427. doi: 10.1016/j. neuroimage.2013.02.063. 
Bassett DS, Bullmore E, Verchinski BA, Mattay VS, Weinberger DR, Meyer-Lindenberg A. 2008. Hierarchical organization of human cortical networks in health and schizophrenia. The Journal of Neuroscience 28:9239-9248. doi: 10.1523/JNEUROSCI.1929-08.2008.

Beckmann CF, Smith SM. 2004. Probabilistic independent component analysis for functional magnetic resonance imaging. IEEE Transactions on Medical Imaging 23:137-152. doi: 10.1109/TMI.2003.822821.

Beckmann CF, Smith SM. 2005. Tensorial extensions of independent component analysis for multisubject FMRI analysis. Neuroimage 25:294-311. doi: 10.1016/j.neuroimage.2004.10.043.

Bellec P, Rosa-Neto P, Lyttelton OC, Benali H, Evans AC. 2010. Multi-level bootstrap analysis of stable clusters in resting-state fMRI. Neuroimage 51:1126-1139. doi: 10.1016/j.neuroimage.2010.02.082.

Bellec P, Lavoie-Courchesne S, Dickinson P, Lerch JP, Zijdenbos AP, Evans AC. 2012. The pipeline system for Octave and Matlab (PSOM): a lightweight scripting framework and execution engine for scientific workflows. Front Neuroinformatics 6:7. doi: 10.3389/fninf.2012.00007.

Berridge KC. 2006. The debate over dopamine's role in reward: the case for incentive salience. Psychopharmacology 191:391-431. doi: 10.1007/s00213-006-0578-x.

Booij J, Knol RJJ. 2007. SPECT imaging of the dopaminergic system in (premotor) Parkinson's disease. Parkinsonism \& Related Disorders 13(Suppl 3):S425-S428. doi: 10.1016/S1353-8020(08)70042-7.

Borghammer P, Østergaard K, Cumming P, Gjedde A, Rodell A, Hall N, Chakravarty MM. 2010. A deformationbased morphometry study of patients with early-stage Parkinson's disease. European Journal of Neurology 17:314-320. doi: 10.1111/j.1468-1331.2009.02807.x.

Braak H, Tredici KD, Rüb U, de Vos RA, Jansen Steur EN, Braak E. 2003. Staging of brain pathology related to sporadic Parkinson's disease. Neurobiology of Aging 24:197-211. doi: 10.1016/S0197-4580(02)00065-9.

Braak H, Ghebremedhin E, Rüb U, Bratzke H, Tredici KD. 2004. Stages in the development of Parkinson's diseaserelated pathology. Cell and Tissue Research 318:121-134. doi: 10.1007/s00441-004-0956-9.

Braak H, Bohl JR, Müller CM, Rüb U, de Vos RAl, Del Tredici K. 2006. Stanley Fahn Lecture 2005: the staging procedure for the inclusion body pathology associated with sporadic Parkinson's disease reconsidered. Movement Disorders 21:2042-2051. doi: 10.1002/mds.21065.

Buckner RL, Sepulcre J, Talukdar T, Krienen FM, Liu H, Hedden T, Andrews-Hanna JR, Sperling RA, Johnson KA. 2009. Cortical hubs revealed by intrinsic functional connectivity: mapping, assessment of stability, and relation to Alzheimer's disease. The Journal of Neuroscience 29:1860-1873. doi: 10.1523/JNEUROSCI.5062-08.2009.

Calhoun VD, Adali T, Pearlson GD, Pekar JJ. 2001. A method for making group inferences from functional MRI data using independent component analysis. Human Brain Mapping 14:140-151. doi: 10.1002/hbm.1048.

Camicioli R. 2013. MR imaging of Parkinson's disease with A cognitive impairment and dementia. Magnetic Resonance Imaging 44.

Carbonell F, Nagano-Saito A, Leyton M, Cisek P, Benkelfat C, He Y, Dagher A. 2014. Dopamine precursor depletion impairs structure and efficiency of resting state brain functional networks. Neuropharmacology 84:90-100. doi: 10.1016/j.neuropharm.2013.12.021.

Chung MK, Worsley KJ, Paus T, Cherif C, Collins DL, Giedd JN, Rapoport JL, Evans AC. 2001. A unified statistical approach to deformation-based morphometry. Neuroimage 14:595-606. doi: 10.1006/nimg.2001.0862.

Collins DL, Evans AC. 1997. Animal: validation and applications of nonlinear registration-based segmentation. International Journal of Pattern Recognition and Artificial Intelligence 11:1271-1294. doi: 10.1142/S0218001497000597.

Collins DL, Neelin P, Peters TM, Evans AC. 1994. Automatic 3D intersubject registration of MR volumetric data. Journal of Computer Assisted Tomography 18:192-205. doi: 10.1097/00004728-199403000-00005.

Coupe P, Yger P, Prima S, Hellier P, Kervrann C, Barillot C. 2008. An optimized blockwise nonlocal means denoising filter for 3-D magnetic resonance images. IEEE Transactions on Medical Imaging 27:425-441. doi: 10. 1109/TMI.2007.906087.

Crossley NA, Mechelli A, Scott J, Carletti F, Fox PT, McGuire P, Bullmore ET. 2014. The hubs of the human connectome are generally implicated in the anatomy of brain disorders. Brain 137:2382-2395. doi: 10.1093/brain/awu132.

Damoiseaux JS, Rombouts S, Barkhof F, Scheltens P, Stam CJ, Smith SM, Beckmann CF. 2006. Consistent restingstate networks across healthy subjects. Proceedings of the National Academy of Sciences of USA 103: 13848-13853. doi: 10.1073/pnas.0601417103.

Daubechies I, Roussos E, Takerkart S, Benharrosh M, Golden C, D’Ardenne K, Richter W, Cohen JD, Haxby J. 2009. Independent component analysis for brain fMRI does not select for independence. Proceedings of the National Academy of Sciences of USA 106:10415-10422. doi: 10.1073/pnas.0903525106.

Diedrichsen J, Balsters JH, Flavell J, Cussans E, Ramnani N. 2009. A probabilistic MR atlas of the human cerebellum. Neuroimage 46:39-46. doi: 10.1016/j.neuroimage.2009.01.045.

Douaud G, Groves AR, Tamnes CK, Westlye LT, Duff EP, Engvig A, Walhovd KB, James A, Gass A, Monsch AU, Matthews PM, Fjell AM, Smith SM, Johansen-Berg H. 2014. A common brain network links development, aging, and vulnerability to disease. Proceedings of the National Academy of Sciences of USA 111:17648-17653. doi: 10.1073/pnas.1410378111.

Duvernoy HM, Bonneville JF, Cabanis EA, Cattin F, Guyot J, Iba-Zizen MT. 1995. The human brain stem and cerebellum: surface, structure, vascularization, and three-dimensional sectional anatomy, with MRI. first edition, Springer. p. 430.

Eidelberg D. 2009. Metabolic brain networks in neurodegenerative disorders: a functional imaging approach. Trends in Neurosciences 32:548-557. doi: 10.1016/j.tins.2009.06.003.

Fearnley JM, Lees AJ. 1991. Ageing and Parkinson's disease: substantia nigra regional selectivity. Brain 114:2283-2301. doi: 10.1093/brain/114.5.2283.

Ford AA, Colon-Perez L, Triplett WT, Gullett JM, Mareci TH, FitzGerald DB. 2013. Imaging white matter in human brainstem. Frontiers in Human Neuroscience 7:400. doi: 10.3389/fnhum.2013.00400. 
Fox MD, Raichle ME. 2007. Spontaneous fluctuations in brain activity observed with functional magnetic resonance imaging. Nature Reviews Neuroscience 8:700-711. doi: 10.1038/nrn2201.

Frost B, Diamond MI. 2010. Prion-like mechanisms in neurodegenerative diseases. Nature Reviews Neuroscience 11:155-159. doi: 10.1038/nrn2786.

Goedert M, Spillantini MG, Del Tredici K, Braak H. 2013. 100 years of Lewy pathology. Nature Reviews Neurology 9:13-24. doi: 10.1038/nrneurol.2012.242.

Goetz CG, Tilley BC, Shaftman SR, Stebbins GT, Fahn S, Martinez-Martin P, Poewe W, Sampaio C, Stern MB, Dodel R, Dubois B, Holloway R, Jankovic J, Kulisevsky J, Lang AE, Lees A, Leurgans S, LeWitt PA, Nyenhuis D, Olanow CW, Rascol O, Schrag A, Teresi JA, van Hilten JJ, LaPelle N, Movement Disorder Society UPDRS Revision Task Force. 2008. Movement Disorder Society-sponsored revision of the Unified Parkinson's Disease Rating Scale (MDS-UPDRS): scale presentation and clinimetric testing results. Movement Disorders 23:2129-2170. doi: 10. 1002/mds.22340.

Greicius MD, Srivastava G, Reiss AL, Menon V. 2004. Default-mode network activity distinguishes Alzheimer's disease from healthy aging: evidence from functional MRI. Proceedings of the National Academy of Sciences of USA 101:4637-4642. doi: 10.1073/pnas.0308627101.

Hammers A, Allom R, Koepp MJ, Free SL, Myers R, Lemieux L, Mitchell TN, Brooks DJ, Duncan JS. 2003. Threedimensional maximum probability atlas of the human brain, with particular reference to the temporal lobe. Human Brain Mapping 19:224-247. doi: 10.1002/hbm.10123.

He Y, Wang J, Wang L, Chen ZJ, Yan C, Yang H, Tang H, Zhu C, Gong Q, Zang Y, Evans AC. 2009. Uncovering intrinsic modular organization of spontaneous brain activity in humans. PLOS ONE 4:e5226. doi: 10.1371/journal. pone.0005226.

Holmqvist S, Chutna O, Bousset L, Aldrin-Kirk P, Li W, Björklund T, Wang ZY, Roybon L, Melki R, Li JY. 2014. Direct evidence of Parkinson pathology spread from the gastrointestinal tract to the brain in rats. Acta Neuropathologica 128:805-820. doi: 10.1007/s00401-014-1343-6.

Hyvärinen A, Karhunen J, Oja E. 2004. Independent component analysis: John Wiley \& Sons.

Ibarretxe-Bilbao N, Junque C, Marti MJ, Tolosa E. 2011. Brain structural MRI correlates of cognitive dysfunctions in Parkinson's disease. Journal of the Neurological Sciences 310:70-74. doi: 10.1016/j.jns.2011.07.054.

Iturria-Medina Y, Canales-Rodríguez EJ, Melie-García L, Valdés-Hernández PA, Martínez-Montes E, AlemánGómez Y, Sánchez-Bornot JM. 2007. Characterizing brain anatomical connections using diffusion weighted MRI and graph theory. Neuroimage 36:645-660. doi: 10.1016/j.neuroimage.2007.02.012.

Iturria-Medina Y, Sotero RC, Toussaint PJ, Evans AC, Alzheimer's Disease Neuroimaging Initiative. 2014. Epidemic spreading model to characterize misfolded proteins propagation in aging and associated neurodegenerative disorders. PLOS Computational Biology 10:e1003956. doi: 10.1371/journal.pcbi.1003956.

Jagust W. 2013. Vulnerable neural systems and the borderland of brain aging and neurodegeneration. Neuron 77:219-234. doi: 10.1016/j.neuron.2013.01.002.

Jucker M, Walker LC. 2013. Self-propagation of pathogenic protein aggregates in neurodegenerative diseases. Nature 501:45-51. doi: 10.1038/nature12481.

Keuken MC, Bazin PL, Crown L, Hootsmans J, Laufer A, Müller-Axt C, Sier R, van der Putten EJ, Schäfer A, Turner R, Forstmann BU. 2014. Quantifying inter-individual anatomical variability in the subcortex using $7 \mathrm{~T}$ structural MRI. Neuroimage 94:40-46. doi: 10.1016/j.neuroimage.2014.03.032.

Luk KC, Kehm V, Carroll J, Zhang B, O'Brien P, Trojanowski JQ, Lee VM. 2012. Pathological $\alpha$-synuclein transmission initiates Parkinson-like neurodegeneration in nontransgenic mice. Science 338:949-953. doi: 10. 1126/science.1227157.

Mai J, Assheuer J, Paxinos G. 2003. Atlas of the human brain. second edition, San Diego: Elsevier.

Marek K, Jennings D, Lasch S, Siderowf A, Tanner C, Simuni T, Coffey C, Kieburtz K, Flagg E, Chowdhury S, Poewe W, Mollenhauer B, Sherer T, Frasier M, Meunier C, Rudolph A, Casaceli C, Seibyl J, Mendick S, Schuff N, Zhang Y, Toga A, Crawford K, Ansbach A, De Blasio P, Piovella M, Trojanowski J, Shaw L, Singleton A, Hawkins K, Eberling J, Brooks D, Russell D, Leary L, Factor S, Sommerfeld B, Hogarth P, Pighetti E, Williams K, Standaert D, Guthrie S, Hauser R, Delgado H, Jankovic J, Hunter C, Stern M, Tran B, Leverenz J, Baca M, Frank S, Thomas CA, Richard I, Deeley C, Rees L, Sprenger F, Lang E, Shill H, Obradov S, Fernandez H, Winters A, Berg D, Gauss K, Galasko D, Fontaine D, Mari Z, Gerstenhaber M, Brooks D, Malloy S, Barone P, Longo K, Comery T, Ravina B, Grachev I, Gallagher K, Collins M, Widnell KL, Ostrowizki S, Fontoura P, La-Roche F, Ho T, Luthman J, van der Brug M, Reith AD, Taylor P. 2011. The parkinson progression marker initiative (PPMI). Progress in Neurobiology 95:629-635. doi: 10.1016/j.pneurobio.2011.09.005.

Masuda-Suzukake M, Nonaka T, Hosokawa M, Oikawa T, Arai T, Akiyama H, Mann DM, Hasegawa M. 2013. Prionlike spreading of pathological $\alpha$-synuclein in. Brain 136:1128-1138. doi: 10.1093/brain/awt037.

Olanow CW, Brundin P. 2013. Parkinson's disease and alpha synuclein: is Parkinson's disease a prion-like disorder? Movement Disorders 28:31-40. doi: 10.1002/mds.25373.

Pereira JB, Svenningsson P, Weintraub D, Brønnick K, Lebedev A, Westman E, Aarsland D. 2014. Initial cognitive decline is associated with cortical thinning in early Parkinson disease. Neurology 82:2017-2025. doi: 10.1212/ WNL.0000000000000483.

Perlbarg V, Bellec P, Anton JL, Pélégrini-Issac M, Doyon J, Benali H. 2007. CORSICA: correction of structured noise in fMRI by automatic identification of ICA components. Magnetic Resonance Imaging 25:35-46. doi: 10. 1016/j.mri.2006.09.042.

Pyatigorskaya N, Gallea C, Garcia-Lorenzo D, Vidailhet M, Lehericy S. 2014. A review of the use of magnetic resonance imaging in Parkinson's disease. Therapeutic Advances in Neurological Disorders 7:206-220. doi: 10. $1177 / 1756285613511507$. 
Raj A, Kuceyeski A, Weiner M. 2012. A network diffusion model of disease progression in dementia. Neuron 73:1204-1215. doi: 10.1016/j.neuron.2011.12.040.

Ross GW, Petrovitch H, Abbott RD, Nelson J, Markesbery W, Davis D, Hardman J, Launer L, Masaki K, Tanner CM, White LR. 2004. Parkinsonian signs and substantia nigra neuron density in decendents elders without PD. Annals of Neurology 56:532-539. doi: 10.1002/ana.20226.

Salamone J, Correa M, Mingote S, Weber S. 2005. Beyond the reward hypothesis: alternative functions of nucleus accumbens dopamine. Current Opinion in Pharmacology 5:34-41. doi: 10.1016/j.coph.2004.09.004.

Scanlon C, Mueller SG, Tosun D, Cheong I, Garcia P, Barakos J, Weiner MW, Laxer KD. 2011. Impact of methodologic choice for automatic detection of different aspects of brain atrophy by using temporal lobe epilepsy as a model. AJNR. American Journal of Neuroradiology 32. doi: 10.3174/ajnr.A2578.

Seeley WW, Crawford RK, Zhou J, Miller BL, Greicius MD. 2009. Neurodegenerative diseases target large-scale human brain networks. Neuron 62:42-52. doi: 10.1016/j.neuron.2009.03.024.

Silbert LC, Kaye J. 2010. Neuroimaging and Cognition in Parkinson's disease dementia. Brain Pathology 20:646-653. doi: 10.1111/j.1750-3639.2009.00368.x.

Sled JG, Zijdenbos AP, Evans AC. 1998. A nonparametric method for automatic correction of intensity nonuniformity in MRI data. IEEE Transactions on Medical Imaging 17:87-97. doi: 10.1109/42.668698.

Smith SM, Jenkinson M, Woolrich MW, Beckmann CF, Behrens TE, Johansen-Berg H, Bannister PR, De Luca M, Drobnjak I, Flitney DE, Niazy RK, Saunders J, Vickers J, Zhang Y, De Stefano N, Brady JM, Matthews PM. 2004. Advances in functional and structural MR image analysis and implementation as FSL. Neuroimage 23:S208-S219. doi: 10.1016/j.neuroimage.2004.07.051.

Smith SM, Fox PT, Miller KL, Glahn DC, Fox PM, Mackay CE, Filippini N, Watkins KE, Toro R, Laird AR, Beckmann CF. 2009. Correspondence of the brain's functional architecture during activation and rest. Proceedings of the National Academy of Sciences of USA 106:13040-13045. doi: 10.1073/pnas.0905267106.

Smith SM, Beckmann CF, Andersson J, Auerbach EJ, Bijsterbosch J, Douaud G, Duff E, Feinberg DA, Griffanti L, Harms MP, Kelly M, Laumann T, Miller KL, Moeller S, Petersen S, Power J, Salimi-Khorshidi G, Snyder AZ, Vu AT, Woolrich MW, Xu J, Yacoub E, Uğurbil K, Van Essen DC, Glasser MF, WU-Minn HCP Consortium. 2013. Restingstate fMRI in the Human Connectome Project. Neuroimage 80:144-168. doi: 10.1016/j.neuroimage.2013.05.039.

Stoodley CJ, Schmahmann JD. 2009. Functional topography in the human cerebellum: a meta-analysis of neuroimaging studies. Neuroimage 44:489-501. doi: 10.1016/j.neuroimage.2008.08.039.

Talairach J, Tournoux P. 1988. Co-planar stereotaxic atlas of the human brain. Stuttgart: Georg Thieme Verlag.

Varentsova A, Zhang S, Arfanakis K. 2014. Development of a high angular resolution diffusion imaging human brain template. Neuroimage 91:177-186. doi: 10.1016/j.neuroimage.2014.01.009.

Warren JD, Rohrer JD, Schott JM, Fox NC, Hardy J, Rossor MN. 2013. Molecular nexopathies: a new paradigm of neurodegenerative disease. Trends in Neurosciences 36:561-569. doi: 10.1016/j.tins.2013.06.007.

Worsley KJ, Liao CH, Aston J, Petre V, Duncan GH, Morales F, Evans AC. 2002. A general statistical analysis for fMRI data. Neuroimage 15:1-15. doi: 10.1006/nimg.2001.0933.

Worsley KJ. 2005. Spatial smoothing of autocorrelations to control the degrees of freedom in fMRI analysis. Neuroimage 26:635-641. doi: 10.1016/j.neuroimage.2005.02.007.

Zhou J, Gennatas ED, Kramer JH, Miller BL, Seeley WW. 2012. Predicting regional neurodegeneration from the healthy brain functional connectome. Neuron 73:1216-1227. doi: 10.1016/j.neuron.2012.03.004.

Zuo XN, Kelly C, Adelstein JS, Klein DF, Castellanos FX, Milham MP. 2010. Reliable intrinsic connectivity networks: test-retest evaluation using ICA and dual regression approach. Neuroimage 49:2163-2177. doi: 10.1016/j. neuroimage.2009.10.080. 